\title{
Conditioning of Quasi-Newton Methods for Function Minimization
}

\author{
By D. F. Shanno
}

\begin{abstract}
Quasi-Newton methods accelerate the steepest-descent technique for function minimization by using computational history to generate a sequence of approximations to the inverse of the Hessian matrix. This paper presents a class of approximating matrices as a function of a scalar parameter. The problem of optimal conditioning of these matrices under an appropriate norm as a function of the scalar parameter is investigated. A set of computational results verifies the superiority of the new methods arising from conditioning considerations to known methods.
\end{abstract}

I. Introduction. Newton's method for minimizing a function $f(x), x$ an $n$-vector, is to generate a sequence of points,

$$
x^{(k+1)}=x^{(k)}-\alpha^{(k)}\left[J^{(k)}\right]^{-1} g^{(k)},
$$

where $g^{(k)}=\nabla F\left(x^{(k)}\right), J^{(k)}=\left[\partial^{2} f / \partial x, \partial x_{j}\right]$, the Hessian matrix of $F$ evaluated at $x^{(k)}$, and $\alpha^{(k)}$ is an appropriately chosen scalar. Quasi-Newton methods use an initial estimate and computational history to generate an estimate $H^{(k)}$ to $\left[J^{(k)}\right]^{-1}$ at each step rather than performing the computational work of evaluating and inverting $J^{(k)}$. The sequence (1) then becomes

$$
x^{(k+1)}=x^{(k)}-\alpha^{(k)} H^{(k)} g^{(k)} .
$$

Here $\alpha^{(k)}$ is chosen to minimize $f$ along $-H^{(k)} g^{(k)}$. Some well-known techniques of this type are the Fletcher-Powell modification of Davidon's method [1], [2], Broyden methods [3], [10], the Barnes-Rosen method [4], [5], and Goldfarb's method [11].

The Fletcher-Powell and Barnes-Rosen methods share the computational feature that, if $f(x)$ is a positive definite quadratic form, the sequence (2) converges in $n$ iterations. This feature is also true of Broyden's method defined in [10], but not of those devised in [3] (see [6]).

Further, the Fletcher-Powell technique guarantees that the matrix, $H^{(k)}$, will always be positive semidefinite, expediting the search for $\alpha^{(k)}$ at each step.

This paper will develop a family of matrices, $H^{(k)}$, as a function of a scalar parameter, $t$, all of which can be shown to possess the quadratic convergence property of the Fletcher-Powell and Barnes-Rosen techniques. It will further be shown that both the Fletcher-Powell and Barnes-Rosen matrices are special cases of this parametric family, and that positivity depends only on proper choice of the parameter.

A problem which arises in connection with quasi-Newton methods occurs when the smallest eigenvalue of $H^{(k)}$ goes to zero. This is the so-called conditioning problem.

Received March 14, 1969, revised January 22, 1970.

AMS Subject Classifications. Primary 6530; Secondary 6550, 9058.

Key Words and Phrases. Function minimization, quasi-Newton methods, variable metric methods, gradient search, steepest-descent methods, stability of search methods, conditioning of search methods, Hessian matrix, inverse approximations.

Copyright $\odot$ 1971, American Mathematical Society 
This paper will investigate the conditioning of the family of matrices as a function of the scalar parameter, and derive an explicit formulation for the best conditioned matrix under an appropriate norm at each step.

Finally, a series of computational results showing marked superiority of alternative choices of the scalar parameter to the Fletcher-Powell and Barnes-Rosen choices will be given.

II. Generation of Approximating Matrices. The technique for generating a series of approximations, $H^{(k)}$, to the inverse of the Hessian at the points, $x^{(k)}$, can be described as follows:

Assume $f(x)$ is a positive definite quadratic form, $H^{(k)}$ the current approximation to the inverse of the Hessian, and $J^{(k)}$ the approximation to the Hessian (note that here the Hessian is a constant matrix). Then if $J^{(k)}$ is an exact approximation,

$$
g^{(k+1)}=g^{(k)}+J^{(k)} \sigma^{(k)},
$$

where again $g(x)=\nabla F(x), \sigma^{(k)}=-\alpha^{(k)} H^{(k)} g^{(k)}$, and $x^{(k+1)}=x^{(k)}+\sigma^{(k)}$. Here again $\alpha^{(k)}$ is chosen to minimize $f$ along $-H^{(k)} g^{(k)}$. Multiplying (3) by $H^{(k)}$, we obtain

$$
H^{(k)} g^{(k+1)}=H^{(k)} g^{(k)}+\sigma^{(k)} .
$$

Let $y^{(k)}=g^{(k+1)}-g^{(k)}$, and (4) becomes

$$
H^{(k)} y^{(k)}=\sigma^{(k)} .
$$

Since (5) in general will not be satisfied, assume the error lies in $H^{(k)}$ rather than in the assumption that $f(x)$ is a quadratic form. We then correct $H^{(k)}$ by

$$
\left(H^{(k)}+D^{(k)}\right) y^{(k)}=\sigma^{(k)},
$$

or

$$
D^{(k)} y^{(k)}=\sigma^{(k)}-H^{(k)} y^{(k)} .
$$

If we let $H^{(k+1)}=H^{(k)}+D^{(k)}$, and $D^{(k)}$ is chosen to satisfy (7), we then have

$$
H^{(k+1)} y^{(k)}=\sigma^{(k)} \text {. }
$$

The algorithm for determining a minimum of $f(x)$ can now be described as follows: Choose initial estimates $x^{(0)}, H^{(0)}$. Let $s^{(k)}=-H^{(k)} g^{(k)}$. Find $\alpha^{(k)} \ni f\left(x^{(k)}-\alpha^{(k)} s^{(k)}\right)$ is a minimum. Note that a necessary condition for this is that $g^{(k+1)^{\prime}} H^{(k)} g^{(k)}=0$.

Let $\sigma^{(k)}=\alpha^{(k)} s^{(k)}$, and $x^{(k+1)}=x^{(k)}+\sigma^{(k)}$. Select a $D^{(k)}$ satisfying (7), and set $H^{(k+1)}=H^{(k)}+D^{(k)}$. Repeat the process until a minimum is obtained. It remains to select the matrix $D^{(k)}$.

A crucial point in the above algorithm is forcing $D^{(k)}$ to satisfy (7), for this guarantees the validity of (8). The importance of this can be seen from

THEOREM 1. If $f(x)$ is a positive definite quadratic form, and at each step (8) is satisfied, then the minimum of $f(x)$ will be reached in at most $n$ iterations.

Proof. The proof is identical to the proof of convergence in the quadratic case of Fletcher and Powell [1], which depends only on the positive definiteness of $f(x)$, the fact that at each step (8) is satisfied, the optimal choice of $\alpha$ at each step, the choice of $\sigma^{(k)}$ as $-\alpha^{(k)} H^{(k)} g^{(k)}$, and choosing $D^{(k)}$ to satisfy (7) in the way shown in Section III. 
III. Selecting the Matrix $D^{(k)}$. In the previous section, we stated that the selection of the matrix, $D^{(k)}$ to satisfy (7) generates a sequence with the desired finite convergence property when $f(x)$ is a positive definite quadratic form. Taking into account the symmetry of the true Hessian, the Barnes-Rosen choice is

$$
D^{(k)}=\frac{\left(\sigma^{(k)}-H^{(k)} y^{(k)}\right)\left(\sigma^{(k)}-H^{(k)} y^{(k)}\right)^{\prime}}{\left(\sigma^{(k)}-H^{(k)} y^{(k)}\right)^{\prime} y^{(k)}} .
$$

This is a correction of rank one as the matrix obviously has only one nonzero eigenvalue.

The Fletcher-Powell choice of $D^{(k)}$ is a rank-two correction,

$$
D^{(k)}=\frac{\sigma^{(k)} \sigma^{(k)}}{\sigma^{(k)} y^{(k)}}-\frac{H^{(k)} y^{(k)} y^{(k) \prime} H^{(k)}}{y^{(k)^{\prime}} H^{(k)} y^{(k)}} .
$$

Hence both matrices satisfy Eq. (7), the Barnes-Rosen considering the right-hand side as a single vector, and the Fletcher-Powell considering the vectors $\sigma^{(k)}$ and $H^{(k)} y^{(k)}$ separately.

A parametric separation of (7) is

$$
D^{(k)} y^{(k)}=t \sigma^{(k)}+(1-t) \sigma^{(k)}-H^{(k)} y^{(k)},
$$

which with proper grouping then yields the solution,

$$
D^{(k)}=t \frac{\sigma^{(k)} \sigma^{(k)}}{\sigma^{(k)} y^{(k)}}+\frac{\left((1-t) \sigma^{(k)}-H^{(k)} y^{(k)}\right)\left((1-t) \sigma^{(k)}-H^{(k)} y^{(k)}\right)^{\prime}}{\left((1-t) \sigma^{(k)}-H^{(k)} y^{(k)}\right)^{\prime} y^{(k)}}
$$

Then $t=0$ is the Barnes-Rosen choice, and $t=1$ the Fletcher-Powell choice. Other parametric separations are possible, and have been developed by Broyden [10] and Goldfarb [11]. It is trivial to show this selection quarantees the validity of Theorem 1 for any value of $t$.

In order to gain insight into the significance of the parameter, $t$, we must first note a weakness of the Barnes-Rosen correction. Suppose at some iteration $k, \alpha^{(k)}=1$. We then have

$$
\sigma^{(k)}-H^{(k)} y^{(k)}=-H^{(k)} g^{(k)}+H^{(k)} g^{(k+1)}+H^{(k)} g^{(k)}=-H^{(k)} g^{(k+1)},
$$

and (9) becomes

$$
D^{(k)}=-\frac{H^{(k)} g^{(k+1)} g^{(k+1)^{\prime}} H^{(k)}}{g^{(k+1)^{\prime}} H^{(k)} g^{(k+1)}} .
$$

Computing, we find

$$
H^{(k+1)} g^{(k-1)}=H^{(k)} g^{(k+1)}+D^{(k)} g^{(k+1)} \equiv 0,
$$

independent of the magnitude of $g^{(k+1)}$. Hence the search vector vanishes when $g^{(k+1)} \neq 0$, forcing a restart of the generation of the approximation to the Hessian.

Now consider the problem of scaling the matrix, $H^{(k)}$, so that the eigenvalue of $H^{(k)}$ in the direction of $\sigma^{(k)}$ is altered, while eigenvalues in all directions orthogonal to $\sigma^{(k)}$ are unchanged. This is accomplished by

$$
\hat{H}^{(k)}=H^{(k)}+t \frac{\sigma^{(k)} \sigma^{(k)}}{\sigma^{(k)^{\prime}} y^{(k)}} .
$$


Solving (16) for $t$ which yields $\hat{\alpha}^{(k)}=1$ gives the solution

$$
t=\alpha^{(k)}-1 / \alpha^{(k)} \text {. }
$$

The formula (16) in a slightly different version was proposed by Davidon [2] with $t=1$ to double the length of $s^{(k)}$ when $\alpha>1$ was indicated. The idea has apparently been carried no further in the modifications of his original work.

We may now consider the generation of rank-two correction matrices as the composite sequence,

$$
\begin{aligned}
\hat{H}^{(k)} & =H^{(k)}+t \frac{\sigma^{(k)} \sigma^{(k)}}{\sigma^{(k)} y^{(k)}}, \\
H^{(k+1)} & =\hat{H}^{(k)}+\frac{\left(\sigma^{(k)}-\hat{H}^{(k)} y^{(k)}\right)\left(\sigma^{(k)}-\hat{H}^{(k)} y^{(k)}\right)^{\prime}}{\left(\sigma^{(k)}-\hat{H}^{(k)} y^{(k)}\right)^{\prime} y^{(k)}} .
\end{aligned}
$$

Computation shows that the composition defined by (18) is identical to (12) for any choice of $t$, yielding

$$
H^{(k+1)}=H^{(k)}+t \frac{\sigma^{(k)} \sigma^{(k)}}{\sigma^{(k)} y^{(k)}}+\frac{\left((1-t) \sigma^{(k)}-H^{(k)} y^{(k)}\right)\left((1-t) \sigma^{(k)}-H^{(k)} y^{(k)}\right)^{\prime}}{\left((1-t) \sigma^{(k)}-H^{(k)} y^{(k)}\right)^{\prime} y^{(k)}}
$$

From the above discussion of singularity in the Barnes-Rosen technique, it is obvious that a choice of $t$ should be restricted to $t \neq\left(\alpha^{(k)}-1\right) / \alpha^{(k)}$.

To further restrict the choice of $t$, we first prove

THEOREM 2. If $H^{(k)}$ is positive definite, $\forall t>\left(\alpha^{(k)}-1\right) / \alpha^{(k)}, H^{(k+1)}$ is positive definite.

Proof. Let $J^{(k)}=\left[H^{(k)}\right]^{-1}, J^{(k+1)}=\left[H^{(k+1)}\right]^{-1}$. Now computation verifies the well-known formula that if $A=B^{-1}, A, B$ symmetric matrices, $\rho$ a scalar, and $z$ an arbitrary vector, then

$$
\left[B+\rho z z^{\prime}\right]^{-1}=A-\frac{\rho}{1+\rho z^{\prime} A z} A z z^{\prime} A .
$$

Applying (20) twice sequentially to (19) yields

$$
\begin{aligned}
J^{(k+1)}= & J^{(k)}+\frac{\alpha^{(k)} t}{1+\alpha^{(k)} t^{2}} \alpha^{(k)} \frac{g^{(k)} g^{(k)}}{g^{(k)} \sigma^{(k)}} \\
& +\frac{\left(\frac{\alpha^{(k)}}{1+\alpha^{(k)} t} g^{(k)}+y^{(k)}\right)\left(\frac{\alpha^{(k)}}{1+\alpha^{(k)} t^{\prime}} g^{(k)}+y^{(k)}\right)^{\prime}}{\left(\frac{\alpha^{(k)}}{\left.1+\alpha^{(k)} t^{(k)}+y^{(k)}\right)^{\prime} \sigma^{(k)}} .\right.} .
\end{aligned}
$$

We first note that for $t>\left(\alpha^{(k)}-1\right) / \alpha^{(k)}, \alpha^{(k)} /\left(1+\alpha^{(k)} t\right)<1$, and hence

$$
\begin{aligned}
\left(\frac{\alpha^{(k)}}{1+\alpha^{(k)} t} g^{(k)}+y^{(k)}\right) & =\left(\frac{\alpha^{(k)}}{1+\alpha^{(k)} t} g^{(k)}-g^{(k)}+g^{(k+1)}\right) \\
& =\left(r g^{(k)}+g^{(k+1)}\right),
\end{aligned}
$$

where $r<0$. Using the condition that $\alpha$ be optimally chosen, we then obtain

$$
\left(r g^{(k)}+g^{(k+1)}\right)^{\prime} \sigma^{(k)}=r g^{(k)} \sigma^{(k)}=-r g^{(k) '} H^{(k)} g^{(k)}>0
$$


by the positive definiteness of $H^{(k)}$. Thus the second correction matrix in (19) is positive semidefinite. We now consider the matrix,

$$
A^{(k)}=J^{(k)}+\frac{\alpha^{(k)} t}{1+\alpha^{(k)} t} \alpha^{(k)} \frac{g^{(k)} g^{(k)}}{g^{(k)} \sigma^{(k)}} .
$$

Note that since

$$
g^{(k) \prime} \sigma^{(k)}=-\alpha^{(k)} g^{(k) \prime} H^{(k)} g^{(k)}, \quad g^{(k) \prime} \sigma^{(k)}<0 .
$$

Hence if $\alpha^{(k)} t /\left(1+\alpha^{(k)} t\right)<0, A^{(k)}$ is trivially positive definite as it is the sum of a positive definite and positive semidefinite matrix.

We now consider the case where $\alpha^{(k)} t /\left(1+\alpha^{(k)} t\right)>0$. Let $z$ be an arbitrary vector, then

$$
\begin{aligned}
z^{\prime} A_{z}^{(k)} z & =z^{\prime} J^{(k)} z+\frac{\alpha^{(k)} t}{1+\alpha^{(k)} t} \alpha^{(k)} \frac{z^{\prime} g^{(k)} g^{(k)} z}{g^{(k)} \sigma^{(k)}} \\
& =\frac{1}{1+\alpha^{(k)} t} z^{\prime} J^{(k)} z+\frac{\alpha^{(k)} t}{1+\alpha^{(k)} t}\left[z^{\prime} J^{(k)} z+\alpha^{(k)} \frac{\left(g^{(k)} z\right)^{2}}{g^{(k)} \sigma^{(k)}}\right] \\
& =\frac{1}{1+\alpha^{(k)} t} z^{\prime} J^{(k)} z+\frac{\alpha^{(k)} t}{1+\alpha^{(k)} t}\left[z^{\prime} J^{(k)} z+\frac{\left(g^{(k)} z\right)^{2}}{g^{(k)} s^{(k)}}\right] .
\end{aligned}
$$

Using the definition of $s^{(k)}$, we see that

$$
z^{\prime} J^{(k)} z+\frac{\left(g^{(k)} z\right)^{2}}{g^{(k)}{ }^{\prime} s^{(k)}}=\frac{z^{\prime} J^{(k)} z g^{(k)} H^{(k)} g^{(k)}-\left(g^{(k)} z\right)^{2}}{g^{(k)^{\prime}} H^{(k)} g^{(k)}} .
$$

Now, since $H^{(k)} J^{(k)}=I$ and $H^{(k)}$ is positive definite, $H^{(k)}$ has a positive square root, as does $J^{(k)}$, and the product of these square roots is again the identity matrix. Hence, letting $T^{(k)}$ be the square root of $H^{(k)}, M^{(k)}$ the square root of $J^{(k)}$, we can rewrite (25) as

$$
\begin{aligned}
z^{\prime} J^{(k)} z+ & \frac{\left(g^{(k)} z\right)^{2}}{g^{(k)} s^{(k)}} \\
& =\frac{\left(M^{(k)} z\right)^{\prime}\left(M^{(k)} z\right)\left(T^{(k)} g^{(k)}\right)^{\prime}\left(T^{(k)} g^{(k)}\right)-\left(\left(T^{(k)} g^{(k)}\right)^{\prime}\left(M^{(k)} z\right)\right)^{2}}{g^{(k)} H^{(k)} g^{(k)}} \geqq 0,
\end{aligned}
$$

with the final inequality following from the Schwartz inequality. Thus $J^{(k+1)}$ is positive definite, and so $H^{(k+1)}$ is positive definite, and for $t=\left(\alpha^{(k)}-1\right) / \alpha^{(k)}, H^{(k+1)}$ is singular.

Since for $t=\left(\alpha^{(k)}-1\right) / \alpha^{(k)}-\epsilon, \epsilon>0$ and small, $g^{(k+1) \prime} H^{(k+1)} g^{(k+1)}<0$, the range of $t$ for this study was restricted to $t>\left(\alpha^{(k)}-1\right) / \alpha^{(k)}$. The next section will deal with the conditioning of the matrix $H^{(k+1)}$ as a function of $t$ in this range.

IV. Conditioning the Matrix $H^{(k+1)}$. As previously noted, computational difficulties arise when the smallest eigenvalue of $H^{(k+1)}$ becomes zero. The arguments of the preceding section show that for $t>(\alpha-1) / \alpha, H^{(k+1)}$ is positive definite, hence, at no finite step does the smallest eigenvalue ever become zero. However, it is possible that if $\lambda_{1}$ is the smallest eigenvalue of $H^{(k+1)}, \lim _{k \rightarrow \infty} \lambda_{1}=0$.

In this case, the iterative technique will degenerate as $k \rightarrow \infty$. To attempt to alleviate this difficulty, we may, at each step, choose $t$ in such a way as to maximize 
the smallest eigenvalue of $H^{(k+1)}$. This is accomplished by choosing $t$ to maximize $z^{\prime} H^{(k+1)} z$ for any arbitrary vector $z$.

A computationally better method of conditioning a matrix, $A$, is to minimize the condition number of $A$, where, in general, the condition number, $\mu$, of a matrix, $A$, is defined to be $\mu=\|A\| \| A^{-1} \mid$. If $\|\cdot\|$ is the spectral norm, and $A$ is symmetric positive semidefinite, then $\mu=\lambda_{n} / \lambda_{1}$, where $\lambda_{n}$ is the largest eigenvalue of $A$. This is not considered here, but is discussed in detail in [12].

To establish that $t$ which maximizes $z^{\prime} H^{(k+1)} z$, we first need the following lemma.

LEMMA 1. For $t>\left(\alpha^{(k)}-1\right) / \alpha^{(k)}, g^{(k+1) '} H^{(k+1)} g^{(k+1)}$ is a monotonically increasing function of $t$, and $\lim _{t \rightarrow \infty} g^{(k+1)^{\prime}} H^{(k+1)} g^{(k+1)}=g^{(k+1)^{\prime}} H^{(k)} g^{(k+1)}$.

Proof. From (8), $\boldsymbol{H}^{(k+1)} y^{(k)}=\sigma^{(k)}$. Hence,

$$
g^{(k+1)^{\prime}} H^{(k+1)} y^{(k)}=\sigma^{(k) \prime} g^{(k+1)}=0,
$$

and we have

$$
g^{(k+1)^{\prime}} H^{(k+1)} g^{(k+1)}-g^{(k+1)^{\prime}} H^{(k+1)} g^{(k)}=0,
$$

yielding

$$
g^{(k+1)^{\prime}} H^{(k+1)} g^{(k+1)}=g^{(k) \prime} H^{(k+1)} g^{(k+1)} .
$$

Applying (29) to (19), and recalling that $\sigma^{(k) '} g^{(k+1)}=-\alpha g^{(k+1)^{\prime}} H^{(k)} g^{(k)}=0$, we have

$$
\begin{aligned}
& g^{(k+1)^{\prime}} H^{(k+1)} g^{(k+1)} \\
& \quad=\frac{g^{(k) \prime}\left((1-t) \sigma^{(k)}-H^{(k)} y^{(k)}\right)\left((1-t) \sigma^{(k)}-H^{(k)} y^{(k)}\right)^{\prime} g^{(k+1)}}{\left((1-t) \sigma^{(k)}-H^{(k)} y^{(k)}\right)^{\prime} y^{(k)}} .
\end{aligned}
$$

Substitution and computation then yields

$$
g^{(k+1)^{\prime}} H^{(k+1)} g^{(k+1)}=\frac{\left(\alpha^{(k)} t+1-\alpha^{(k)}\right) g^{(k) \prime} H^{(k)} g^{(k)} g^{(k+1)^{\prime}} H^{(k)} g^{(k+1)}}{\left(\alpha^{(k)} t+1-\alpha^{(k)}\right) g^{(k)^{\prime}} H^{(k)} g^{(k)}+g^{(k+1)^{\prime}} H^{(k)} g^{(k+1)}}
$$

Differentiating (31) with respect to $t$ yields

$$
\begin{aligned}
& \frac{d g^{(k+1)^{\prime}} H^{(k+1)} g^{(k+1)}}{d t} \\
& \quad=\frac{\alpha^{(k)} g^{(k)^{\prime}} H^{(k)} g^{(k)}\left(g^{(k+1)^{\prime}} H^{(k)} g^{(k+1)}\right)^{2}}{\left(\left(\alpha^{(k)} t+1-\alpha^{(k)}\right) g^{(k)} H^{(k)} g^{(k)}+g^{(k+1)^{\prime}} H^{(k)} g^{(k+1)}\right)^{2}}>0 .
\end{aligned}
$$

Thus $g^{(k+1)^{\prime}} H^{(k+1)} g^{(k+1)}$ is a monotonically increasing function of $t$, and from (31), $\lim _{t \rightarrow \infty} g^{(k+1)^{\prime}} H^{(k+1)} g^{(k+1)}=g^{(k+1)^{\prime}} H^{(k)} g^{(k+1)}$, and the lemma is proved.

We may now show

THEOREM 3. Let $z$ be an arbitrary vector. Then $z^{\prime} H^{(k+1)} z$ is a nondecreasing function of $t$, for $t>\left(\alpha^{(k)}-1\right) / \alpha^{(k)}$.

Proof. Since $H^{(k)}$ is positive definite, and $g^{(k+1) \prime} H^{(k)} g^{(k)}=0, \exists$ a basis for $E^{n}$ composed of $g^{(k)}, g^{(k+1)}$, and $n-2$ vectors $\xi_{1}, \cdots, \xi_{n-2}$ which are mutually $H^{(k)}$ orthogonal to $g^{(k)}, g^{(k+1)}$ and to each other. Now since $\xi_{i}^{\prime} H^{(k)} g^{(k)}=\xi_{i}^{\prime} H^{(k)} g^{(k+1)}=0$, trivially $\xi_{i}^{\prime} H^{(k+1)} \xi_{j}=0$ for $i \neq j$. Further, $\xi_{i}^{\prime} H^{(k+1)} g^{(k+1)}=\xi_{i}^{\prime} H^{(k+1)} g^{(k)}=0$, and $\xi_{i}^{\prime} H^{(k+1)} \xi_{i}$ is independent of $t$. All of these may easily be verified by using (19). Finally, since $\xi_{i}^{\prime} H^{(k+1)} g^{(k+1)}=\xi_{i}^{\prime} H^{(k+1)} g^{(k)}=0$, we have

$$
\xi_{i}^{\prime} H^{(k+1)} g^{(k+1)}-\xi_{i}^{\prime} H^{(k+1)} g^{(k)}=\xi_{i}^{\prime} H^{(k+1)} y^{(k)}=0 \text {. }
$$


Further, since for $t>\left(\alpha^{(k)}-1\right) / \alpha^{(k)}, H^{(k+1)}$ is positive definite, and since $g^{(k+1) \prime} H^{(k+1)} y^{(k)}=0$, the $\xi_{2}$ 's, together with $g^{(k+1)}$ and $y^{(k)}$, form a basis for $E^{n}$. Hence, let

$$
z=\sum_{i=1}^{n-2} a_{i} \xi_{i}+a_{n-1} g^{(k+1)}+a_{n} y^{(k)} .
$$

Then

$$
z^{\prime} H^{(k+1)} z=\sum_{i=1}^{n-2} a_{i}^{2} \xi_{i}^{\prime} H^{(k+1)} \xi_{i}+a_{n-1}^{2} g^{(k+1)^{\prime}} H^{(k+1)} g^{(k+1)}+a_{n}^{2} y^{(k) \prime} H^{(k+1)} y^{(k) \prime},
$$

where

$$
\sum_{i=1}^{n-2} a_{i}^{2} \xi_{i}^{\prime} H^{(k+1)} \xi_{i}=\sum_{i=1}^{n-2} a_{i}^{2} \xi_{i}^{\prime} H^{(k)} \xi_{i}
$$

and, hence, is independent of $t$. We now note that

$$
y^{(k) '} H^{(k+1)} y^{(k)}=\sigma^{(k)^{\prime}} y^{(k)}=\alpha^{(k)} g^{(k) '} H^{(k)} g^{(k)},
$$

independent of $t$. Hence,

$$
\frac{d z^{\prime} H^{(k+1)} z}{d t}=a_{n-1}^{2} \frac{d g^{(k+1)^{\prime}} H^{(k+1)} g^{(k+1)}}{d t},
$$

and, by Lemma 1 , this is greater than 0 if $a_{n-1} \neq 0$. This proves the theorem.

We have thus shown that the condition of $H^{(k+1)}$ improves monotonically with $t$. This necessitates finding a closed form representation of $H^{(k+1)}$ for $t=\infty$.

THEOREM 4. Let $H^{(k+1)}$ be defined by (19), and let

Then

$$
r=\frac{\sigma^{(k)} y^{(k)}}{\sigma^{(k)} y^{(k)}+y^{(k) '} H^{(k)} y^{(k)}} .
$$

$\lim H^{(k+1)}$

$$
=H^{(k)}+\frac{\left(\sigma^{(k)}-r H^{(k)} y^{(k)}\right)\left(\sigma^{(k)}-r H^{(k)} y^{(k)}\right)^{\prime}}{\left(\sigma^{(k)}-r H^{(k)} y^{(k)}\right)^{\prime} y^{(k)}}+(r-1) \frac{H^{(k)} y^{(k)} y^{(k)} H^{(k)}}{y^{(k)} H^{(k)} y^{(k)}} .
$$

Proof. As in Theorem 2, apply (20) sequentially to (19), again yielding

$$
\begin{aligned}
& J^{(k+1)}=J^{(k)}+\frac{\alpha^{(k)} t}{1+\alpha^{(k)} t} \alpha^{(k)} \frac{g^{(k)} g^{(k)}}{g^{(k)} \sigma^{(k)}} \\
&+\frac{\left(\frac{\alpha^{(k)}}{1+\alpha^{(k)} t} g^{(k)}+y^{(k)}\right)\left(\frac{\alpha^{(k)}}{1+\alpha^{(k)} t} g^{(k)}+y^{(k)}\right)^{\prime}}{\left(\frac{\alpha^{(k)}}{1+\alpha^{(k)} t} g^{(k)}+y^{(k)}\right)^{\prime} \sigma^{(k)}} .
\end{aligned}
$$

Then

$$
\lim _{t \rightarrow \infty} J^{(k+1)}=J^{(k)}+\alpha^{(k)} \frac{g^{(k)} g^{(k)} \cdot}{g^{(k)} \sigma^{(k)}}+\frac{y^{(k)} y^{(k)}}{y^{(k)} \sigma^{(k)}} .
$$

Now apply (20) to

$$
S^{(k)}=\left[J^{(k)}+\frac{y^{(k)} y^{(k)}}{\sigma^{(k)} y^{(k)}}\right],
$$




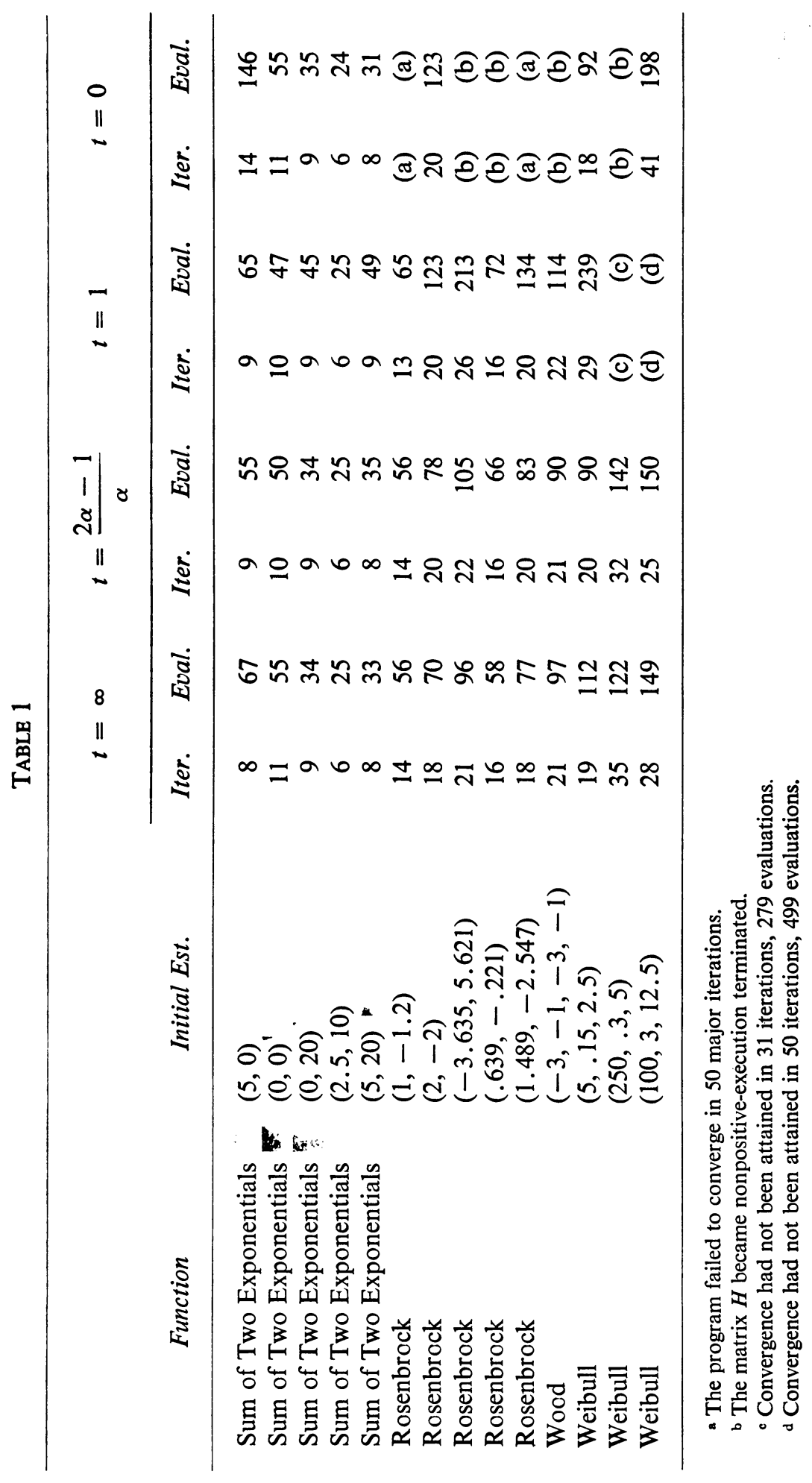


then again to

$$
J^{(k+1)}=S^{(k)}+\alpha^{(k)} \frac{g^{(k)} g^{(k)}}{g^{(k)^{\prime}} \sigma^{(k)}},
$$

and the result follows.

It should be noted that this representation for $H^{(k+1)}$ has been derived by Goldfarb [11] from other considerations.

V. Discussion. Section II showed that matrices of the form (19) have the desired convergence property for all values of $t$. Section III showed that the desired positive definiteness is maintained for all $t>\left(\alpha^{(k)}-1\right) / \alpha^{(k)}$. We first note that this is a reverification of the Fletcher-Powell proof of stability of $H^{(k)}$, for $1>\left(\alpha^{(k)}-1\right) / \alpha^{(k)}$ for all $\alpha^{(k)}$. However, this also points out the weakness of the Fletcher-Powell method, for, as $\alpha^{(k)}$ becomes large, $H^{(k+1)}$ becomes ill-conditioned, slowing the process of convergence. Section IV shows that $t=\infty$ yields maximum stability. However, it is not necessarily the case that this provides convergence to the desired optimum in the minimum number of iterations. To this end, it may prove optimal to choose $t$ at each step as a function of $\alpha^{(k)}$. To accomplish this, the relation, $t^{(k)}=\left(2 \alpha^{(k)}-1\right) / \alpha^{(k)}$, was tried computationally along with $t=\infty, t=1$, and $t=0$, on a variety of problems. The results are documented in Section VI.

Finally, note that $t^{(k)}=\left(2 \alpha^{(k)}-1\right) / \alpha^{(k)}$ satisfies $t^{(k)}>\left(\alpha^{(k)}-1\right) / \alpha^{(k)}$. Also, $t^{(k)}=$ $\left(2 \alpha^{(k)}-1\right) / \alpha^{(k)}$ has the significance of being the value of $t^{(k)}$ obtained by optimally scaling

$$
\hat{H}^{(k)}=H^{(k)}+\frac{\alpha^{(k)}-1}{\alpha^{(k)}} \frac{\sigma^{(k)} \sigma^{(k)}}{\sigma^{(k)} y^{(k)}},
$$

and then updating, using the Fletcher-Powell-Davidon technique, substituting $\hat{H}^{(k)}$ for $H^{(k)}$ and giving

$$
H^{(k+1)}=\hat{H}^{(k)}+\frac{\sigma^{(k)} \sigma^{(k)} \cdot}{\sigma^{(k)} y^{(k)}}-\frac{\hat{H}^{(k)} y^{(k)} y^{(k)} H^{(k)}}{y^{(k)} H^{(k)} y^{(k)}} .
$$

VI. Computational Results. The methods corresponding to $t=\infty, t=$ $\left(2 \alpha^{(k)}-1\right) / \alpha^{(k)}, t=1$, and $t=0$, were tested for various initial estimates on four functions. They are the sum of two exponentials documented by Box [7], and defined by

$$
f\left(x_{1}, x_{2}\right)=\sum_{i=1}^{10}\left[\left(e^{-x_{1} t_{i}}-e^{-x_{2} t_{i}}\right)-\left(e^{-t_{i}}-e^{-10 t_{i}}\right)\right]^{2} .
$$

where $t_{i}$ ranges from .1 to 1 in steps of .1 ; Rosenbrock's function with the initial estimates suggested by Leon [8], and defined by

$$
f\left(x_{1}, x_{2}\right)=100\left(x_{2}-x_{1}^{2}\right)^{2}+\left(1-x_{1}\right)^{2} ;
$$

Wood's function as documented by Pearson [9], and defined by

$$
\begin{aligned}
f\left(x_{1}, x_{2}, x_{3}, x_{4}\right)= & 100\left(x_{2}-x_{1}^{2}\right)^{2}+\left(1-x_{1}\right)^{2}+90\left(x_{4}-x_{3}^{2}\right)^{2}+\left(1-x_{3}\right)^{2} \\
& +10.1\left[\left(x_{2}-1\right)^{2}+\left(x_{4}-1\right)^{2}\right]+19.8\left(x_{2}-1\right)\left(x_{4}-1\right) ;
\end{aligned}
$$


and finally the Weibull function, defined by

$$
f\left(x_{1}, x_{2}, x_{3}\right)=\sum_{i=1}^{99}\left(\exp \left(-\frac{1}{x_{1}}\left(t_{i}-x_{3}\right)^{x_{2}}\right)-y_{i}\right)^{2},
$$

where the $y^{(i)}$ and $t^{(i)}$ are perfect data generated for the 99 points corresponding to $y=.1$ to .99 , in steps of .01 , for the values $x_{1}=50, x_{2}=1.5, x_{3}=25$. The initial estimates are those suggested by Dale Fimple.

Box's three-parameter exponential problem was also tried, but nonuniqueness of the optimum caused different methods to converge to different optima, invalidating comparisons.

In all cases, convergence was determined when $\left|\sigma_{i}^{(k)}\right| \leqq 10^{-5}\left|x_{i}^{(k)}\right|$ and $\left|g_{i}^{(k)}\right| \leqq$ $10^{-5}\left|x_{i}^{(k)}\right|$. Further, the search for $\alpha^{(k)}$ terminated when $g^{(k+1) \prime} \sigma^{(k)}<10^{-3} S$, where $S=\sum_{i=1}^{n}\left|g_{i}^{(k+1)}\right|\left|\sigma_{i}^{(k)}\right| / n$. Finally, approximations to $\alpha^{(k)}$ were found by the cubic quadrature devised by Davidon [2]. In the table, the number of iterations is the number of times $H^{(k)}$ was updated, and the number of evaluations, the true number of function evaluations used.

The results in the table make it obvious that the Barnes-Rosen method is quite unstable. Further, in virtually all cases, the $t=\alpha$ and $t=\infty$ methods outperformed $t=1$, and the difference became more notable as the complexity of the function increased.

Finally, the relative performances of $t=\infty$ and $t=\left(2 \alpha^{(k)}-1\right) / \alpha^{(k)}$ are similar enough to indicate that an optimum $\alpha$ sensitive strategy would outperform the maximum conditioning strategy, but perhaps only marginally.

University of Chicago

Chicago, Illinois 60637

1. R. Fletcher \& M. J. D. Powell, "A rapidly convergent descent method for minimization," Comput. J., v. 6, 1963/64, pp. 163-168. MR 27 \#2096.

2. W. C. Davidon, Variable Metric Method for Minimization, Argonne National Laboratory Report ANL-5990, November 1959.

3. C. G. BROYDEN, "A class of methods for solving nonlinear simultaneous equations," Math. Comp., v. 19, 1965, pp. 577-593. MR 33 \#6825.

4. J. G. P. BARNES, "An algorithm for solving non-linear equations based on the secant method," Comput J., v. 8, 1965, pp. 66-72. MR 31 \#5330.

5. E. M. RosEn, "A review of quasi-Newton methods in nonlinear equation solving and unconstrained optimization," Nat. Conference of the ACM, Proceedings of the Twenty-First Conference, Thompson Book Co., Washington, D.C., 1966, pp. 37-41.

6. F. J. ZELEZNIK, "Quasi-Newton methods for nonlinear equations," J. Assoc. Comput. Mach., v. 15, 1968, pp. 265-271. MR 38 \#387.

7. M. J. Box, "A comparison of several current optimization methods, and the use of transformations in constrained problems," Comput. J., v. 9, 1966, pp. 67-77. MR 33 \#870.

8. A. Leon, A Comparison Among Eight Known Optimizing Procedures, Internal Working Paper No. 20, Space Sciences Laboratory, University of California, Berkeley, August 1964.

9. J. D. Pearson, On Variable Metric Methods of Minimization, Research Analysis Corp. Technical Paper, RAC-TP-302, February 1968.

10. C. G. BROYDEN, "Quasi-Newton methods and their application to function minimisation," Math. Comp., v. 21, 1967, pp. 368-381. MR 36 \#7317.

11. D. GoldFARB, "A family of variable-metric methods derived by variational means," Math. Comp., v. 24, 1970, pp. 23-26.

12. D. F. Shanno \& P. C. Ketrler, "Optimal conditioning of quasi-Newton methods," Math. Comp., v. 24, 1970, pp. 657-664. 